\title{
Current approaches to reduce or eliminate mitochondrial DNA mutations
}

\author{
Liang Yang, Tingfang Mei, Xiaobing Lin, Haite Tang, Yi Wu, Rui Wang, Jinglei Liu, \\ Zahir Shah \& Xingguo Liu*

\begin{abstract}
Key Laboratory of Regenerative Biology, Guangdong Provincial Key Laboratory of Stem Cell and Regenerative Medicine, South China Institute for Stem Cell Biology and Regenerative Medicine, Guangzhou Institutes of Biomedicine and Health, Chinese Academy of Sciences,
\end{abstract} \\ Guangzhou 510530, China
}

Received November 25, 2014, accepted December 10, 2015; published online April 21, 2016

Citation: Yang, L., Mei, T., Lin, X., Tang, H., Wu, Y., Wang, R., Liu, J., Shah, Z., and Liu, X. (2016). Current approaches to reduce or eliminate mitochondrial DNA mutations. Sci China Life Sci 59, 532-535. doi: 10.1007/s11427-014-0276-8

Mitochondrial DNA (mtDNA) mutations have been implicated in a broad range of disorders which severely affect human health (Wallace, 1999). Some drugs have been developed to slow down pathological changes of mitochondrial disorders. However, there is no effective treatment for patients with mtDNA mutations. mtDNA is less protected and has fewer repair mechanisms than nuclear DNA (nDNA). Such a reality results in a much higher mutation rate in mtDNA than that in nDNA. The mixture of mutated mtDNA versus wild-type mtDNA is known as heteroplasmy. Mitochondrial threshold effect refers to the fact that mtDNA mutation must accumulate to high proportions $(60 \%-90 \%)$ before respiratory activity is affected (Schon et al., 2012). It is feasible to selectively reduce the levels of mutated mtDNA while sparing wild-type mtDNA to skew this ratio back to a healthier range. Here, we describe the link between mtDNA mutation and mitochondrial diseases, and we summarize several newly developed approaches with regard to the reduction or elimination of mtDNA mutation in mammals. These methods include nuclear gene modulation, molecular approaches targeting mutated mtDNA, mtDNA replacement, and induced pluripotent stem cell (iPSC) modeling. These various methods have their own advantages and limitations.

*Corresponding author (email: liu_xingguo@gibh.ac.cn)
Mitochondria including mtDNA molecules are constantly turned over (i.e. replicated and degraded) to maintain a pool of healthy mitochondria in cells. This process is controlled by mitochondrial fusion-fission dynamics. The defect of mitochondrial fusion can cause a higher mtDNA mutation rate in skeletal muscle. Moreover, the mutations of mtDNA polymerase gamma (POLG) also result in increased spontaneous mtDNA mutation rates. These defects can be rescued by targeting nuclear DNA-expressed counterparts of the affected molecules into mitochondria. Such a process is called "allotropic" strategy (Torchilin et al., 2002). Exogenous genes have been successfully transfected into mitochondria to compensate for the function of mutated mtDNA. However, it can't effectively intervene mutated mtDNA due to the very low transfection efficiency. In some cases, defects were reported to be rescued by this "allotopic" strategy. This approach is limited because imported mitochondrial proteins fail to assemble correctly in respiratory complexes. These methods are ineffective in living cells and lack the flexibility to target clinically relevant mutations. Also they do not produce stable changes in heteroplasmy, nor are they readily translatable to animals.

To reduce the level of heteroplasmy, several approaches were developed to target mutated mtDNA. One approach is to specifically design synthetic peptide nucleic acid molecules to inhibit mutated mtDNA replication (Taylor et al., 1997). This approach has been demonstrated to be effective 
in vitro. Unfortunately, the designed molecules are not able to cross the inner mitochondrial membrane, and as a result, are not capable of interacting with mtDNA in vivo. Another approach uses mitochondrial targeting recombinant RNAs to modulate the rate of mtDNA mutation associated with Kearns Sayre Syndrome (Comte et al., 2013). Although the inhibitory effect was partial $(15 \%-35 \%$ decrease of mutated mtDNA level), it may have a long-term therapeutic interest, because only high levels of mutations in human mtDNA become pathogenic. These findings may be relevant to the development of a new tool for therapy of mtDNA associated diseases. However, these attempts are ineffective in living cells and lack the flexibility to target clinically relevant mutations. Thus, they could not produce stable changes in heteroplasmy or be readily translatable to animals.

Mitochondrial targeting restriction endonucleases can also be used to cleave mutated mtDNA molecules. The transcription activator-like effector nucleases (TALENs) are artificial restriction enzymes generated by fusing a TAL effector DNA binding domain to a DNA cleavage domain. Restriction enzymes are enzymes that cut DNA strands at a specific sequence. Transcription activator-like effectors (TALEs) can be quickly engineered to bind practically any desired DNA sequence. By combining such an engineered TALE with a DNA cleavage domain (which cuts DNA strands), one can engineer restriction enzymes that are specific for any desired DNA sequence. When these restriction enzymes are introduced into mitochondria, they can be used for cleaving different classes of pathogenic mtDNA mutations. Mitochondria-targeted TALEN (mitoTALEN) expression leads to permanent reductions in deletion or point-mutant mtDNA in patient-derived cells, thus raising the possibility that these mitochondrial nucleases can be therapeutic for some mitochondrial diseases. Transient expression of mitoTALENs is sufficient to produce lasting changes in mtDNA heteroplasmy (Bacman et al., 2013). It is therefore reasonable to expect that permanent correction of heteroplasmy levels, potentially rescuing the oxidative phosphorylation deficiency in affected tissues, might be achieved after one or a small number of administrations of mitoTALEN, either as a genetic or protein agent. Given their ability to alter mtDNA heteroplasmy in living cells, the use of nucleases is expanded. MitoTALENs is reengineered as a powerful and flexible platform to reduce the abundance of mutated mtDNA relative to wild-type mtDNA (Sung et al., 2013; Reddy et al., 2015), which shows more efficiency and specificity.

Replacing mutated mtDNA with normal mtDNA is an approach which provides new thoughts for treating mitochondrial diseases. In somatic cells, mesenchymal stem cells (MSCs) have been reported to transfer mitochondria to some specific cells. In germlines, techniques including pronuclear and metaphase II spindle transfer have been report- ed to prevent the transmission of serious mtDNA diseases (Tachibana et al., 2009).

To prevent the passing of mutated mtDNA from parents to offspring, the nuclear genome transfer between patients' and healthy eggs to replace mutated mtDNAs holds promise. Such an approach can totally replace mutated mtDNA with normal mtDNA. A promising recent advancement in assisted reproductive technologies involves the efficient replacement of mutated mtDNA in unfertilized oocytes or zygotes with normal donor mitochondria, a replacement which eliminates the mutated mtDNA which is inherited by the offspring from parents. Before fertilization or implantation, of first importance is determining the mtDNA heteroplasmic genotype of oocytes or embryos. Only those embryos with a desirable mtDNA genotype could be retained for implantation into the mother. Several microsurgical nuclear transfer procedures have been developed for germline genome transfer. Among these procedures are spindle-chromosome transfer (ST), pronuclear transfer (PNT) and polar body transfer.

$\mathrm{ST}$ is conducted at the mature oocyte stage when the nuclear DNA material is assembled into metaphase chromosomes. The spindle is easily isolated and then transplanted into donated enucleated unfertilized oocyte. The reconstructed oocyte with no mutated mtDNA, can be fertilized and subsequently transplanted to the patient. PNT is a method to transfer the nucleus of patient zygote (mutated mtDNA) into an enucleated oocyte or zygote (contains healthy mtDNA). PNT between zygotes, as well as ST, has the potential to prevent the transmission of mtDNA disease in humans. The transfer of polar body is considered as a better method to prevent the transmission of mutated mtDNA because polar body has fewer mitochondria. Embryos reconstructed by polar body transfer support normal fertilization and produce live offspring. Generation from polar body transfer leads to minimal donor mtDNA carryover as compared to the $F_{1}$ generation from other procedures. This indicates that polar body transfer has great potential to prevent inherited mtDNA diseases.

Reproductive options involving mutated mtDNA replacement therapy have the potential to prevent mtDNA disease transmission. Replacing mutated mtDNA by ST or PNT does not involve risky genome modifications with recombinant DNA vectors, and has relevance to all mtDNA mutation types. However, this therapy cannot eliminate these risks completely and the offspring still possess the chances of succombing to mitochondrial diseases (Amato et al., 2014). Polar body transfer that leads to minimal donor mtDNA carryover demonstrates great potential for the prevention of inherited mtDNA diseases (Wang et al., 2014). The current challenge is to chart a course that translates these preclinical and clinical studies into clinical trials that evaluate efficacy and safety in affected couples. It is also 
important to continue ethical and regulatory discussions related not only to germline mtDNA replacement but also to germline gene therapy in general.

Since tissue sources are limited by ethical issues and injury, it is difficult for researchers to study the mitochondrial diseases further or even perform drug screening. Somatic cells can be directly reprogrammed using four transcription factors (Oct4, Sox2, Klf4 and c-Myc) to generate iPSCs. These iPSCs have the capacity to differentiate into any tissue (Yu et al., 2007). Direct reprogramming allows the creation of patient-specific pluripotent cells that retain the cytoplasmic contents of donor cells, including disease-associated mtDNA. IPSCs are particularly amenable for studying mtDNA disorders, as cytoplasmic genetic material is retained during direct reprogramming. The technique can be used to establish iPSC-derived models for disorders with mtDNA mutations, which have opened a remarkable avenue for theoretic study and therapeutic application (Prigione, 2015; Ma et al., 2015). Several studies have revealed that mitochondrial heteroplasmy segregate within iPSC clones derived from patients with mtDNA mutation. Compared to the mtDNA mutation rates of these original cells from patients with mtDNA mutation, these mitochondrial disease-specific iPSC (mt-iPSC) clones derived from patients' cells exhibit a bimodal degree of mutation heteroplasmy. The stable mt-iPSCs with much higher mtDNA mutation rates can be used as a source of cells for human mitochondrial disease modeling in vitro, while those with undetectable mtDNA mutations could provide an unlimited, disease-free supply of cells for autologous transplantation therapy.

Mutation-free iPSCs are a potential source of healthy, autologous tissue-specific progenitors for patients with mitochondrial diseases, although significant hurdles remain in the translation of iPSC-based cellular therapy. This cell-based platform unlocks the distinctive advantages of patient-specific iPSC technology for genotype/phenotype analysis allowing discovery of patient-specific drug targets, mechanisms of mtDNA mutations in the native context of nuclear DNA, and ultimately offers the potential of a curative cell-based therapeutic strategy for regenerative mitochondrial medicine.

The mt-iPSCs that carry varying burdens of mutated mtDNA from patients provide a new tool for in vitro studies of mitochondrial dysfunction. Mt-iPSCs offer a valuable complement to hybrid techniques because disease-carrying and disease-free iPSC lines can be directly differentiated into precise tissue types to illuminate the pathophysiology of mtDNA disorders, and possibly to screen drugs that will support impaired cell-type specific functions.

Overall, mtDNA modification or replacement can be combined with iPSCs technology to prevent mitochondrial damage caused by mtDNA mutations. After mutated
mtDNAs in somatic cells are corrected by mito-TALEN or mtDNA replacement, the somatic cells with less mutated mtDNA can be further reprogrammed to iPSCs for tissue-specific progenitors for patients. In summary, the combination of iPSCs technology with mtDNA replacement holds promise for treating patients with mtDNA mutations.

Compliance and ethics The author(s) declare that they have no conflict of interest.

Acknowledgements This work was supported by the National Basic Research Program of China (2013CB967403, 2012CB721105), the "Strategic Priority Research Program" of the Chinese Academy of Sciences (XDA01020108), the National High-tech R\&D Program (2012AA02A708), the National Natural Science Foundation projects of China (31271527, 81570520), Guangzhou Science and Technology Program (2014Y2-00161), Guangdong Natural Science Foundation for Distinguished Young Scientists (S20120011368), Guangdong Province Science and Technology Innovation Young Talents Program (2014TQ01R559), Guangdong Province Science and Technology Program (2015A020212031), the PhD Start-up Fund of Natural Science Foundation of Guangdong Province (2014A030310071), and the 'One Hundred Talents', Project for Prof. Xingguo Liu from the Chinese Academy of Sciences.

Amato, P., Tachibana, M., Sparman, M., and Mitalipov, S. (2014). Three-parent in vitro fertilization: gene replacement for the prevention of inherited mitochondrial diseases. Fertil Steril 101, 31-35.

Bacman, S.R., Williams, S.L., Pinto, M., Peralta, S., and Moraes, C.T. (2013). Specific elimination of mutant mitochondrial genomes in patient-derived cells by mitoTALENs. Nat Med 19, 1111-1113.

Comte, C., Tonin, Y., Heckel-Mager, A.M., Boucheham, A., Smirnov, A., Aure, K., Lombes, A., Martin, R.P., Entelis, N., and Tarassov, I. (2013). Mitochondrial targeting of recombinant RNAs modulates the level of a heteroplasmic mutation in human mitochondrial DNA associated with Kearns Sayre Syndrome. Nucleic Acids Res 41, 418-433.

Ma, H., Folmes, C.D., Wu, J., Morey, R., Mora-Castilla, S., Ocampo, A., Ma, L., Poulton, J., Wang, X., Ahmed, R., Kang, E., Lee, Y., Hayama, T., Li, Y., Van Dyken, C., Gutierrez, N.M., Tippner-Hedges, R., Koski, A., Mitalipov, N., Amato, P., Wolf, D.P., Huang, T., Terzic, A., Laurent, L.C., Izpisua Belmonte, J.C., and Mitalipov, S. (2015). Metabolic rescue in pluripotent cells from patients with mtDNA disease. Nature 524, 234-238.

Prigione, A. (2015). Induced pluripotent stem cells (iPSCs) for modeling mitochondrial DNA disorders. Methods Mol Biol 1265, 349-356.

Reddy, P., Ocampo, A., Suzuki, K., Luo, J., Bacman, S.R., Williams, S.L., Sugawara, A., Okamura, D., Tsunekawa, Y., Wu, J., Lam, D., Xiong, X., Montserrat, N., Esteban, C.R., Liu, G.H., Sancho-Martinez, I., Manau, D., Civico, S., Cardellach, F., Del Mar O'Callaghan, M., Campistol, J., Zhao, H., Campistol, J.M., Moraes, C.T., and Izpisua Belmonte, J.C. (2015). Selective elimination of mitochondrial mutations in the germline by genome editing. Cell 161, 459-469.

Schon, E.A., DiMauro, S., and Hirano, M. (2012). Human mitochondrial DNA: roles of inherited and somatic mutations. Nat Rev Genet 13, 878-890.

Sung, Y.H., Baek, I.J., Kim, D.H., Jeon, J., Lee, J., Lee, K., Jeong, D., Kim, J.S., and Lee, H.W. (2013). Knockout mice created by TALEN-mediated gene targeting. Nat Biotechnol 31, 23-24.

Tachibana, M., Sparman, M., Sritanaudomchai, H., Ma, H., Clepper, L., Woodward, J., Li, Y., Ramsey, C., Kolotushkina, O., and Mitalipov, S. (2009). Mitochondrial gene replacement in primate offspring and embryonic stem cells. Nature 461, 367-372.

Taylor, R.W., Chinnery, P.F., Turnbull, D.M., and Lightowlers, R.N. 
(1997). Selective inhibition of mutant human mitochondrial DNA replication in vitro by peptide nucleic acids. Nat Genet 15, 212-215.

Torchilin, V.P., Khaw, B.A., and Weissig, V. (2002). Intracellular targets for DNA delivery: nuclei and mitochondria. Somat Cell Mol Genet 27. 49-64.

Wallace, D.C. (1999). Mitochondrial diseases in man and mouse. Science 283, 1482-1488.
Wang, T., Sha, H., Ji, D., Zhang, H.L., Chen, D., Cao, Y., and Zhu, J. (2014). Polar body genome transfer for preventing the transmission of inherited mitochondrial diseases. Cell 157, 1591-1604.

Yu, J., Vodyanik, M.A., Smuga-Otto, K., Antosiewicz-Bourget, J., Frane, J.L., Tian, S., Nie, J., Jonsdottir, G.A., Ruotti, V., Stewart, R., Slukvin, II, and Thomson, J.A. (2007). Induced pluripotent stem cell lines derived from human somatic cells. Science 318, 1917-1920.

Open Access This article is distributed under the terms of the Creative Commons Attribution License which permits any use, distribution, and reproduction in any medium, provided the original author(s) and source are credited. 